\title{
Oxidative Stress as Marker of Sepsis in Onco Hematological Patients: A Pilot Study
}

Fracchiolla $N^{1^{*}}$, Cortelezzi $A^{1}$, Orofino $\mathbf{N}^{1}$, Novembrino $C^{3}$, De Giuseppe $\mathbf{R}^{2}$, Turrini $\mathbf{M}^{4}$ and Bamonti $\mathrm{F}^{2}$

${ }^{1}$ Hematology and Transplantation Unit, IRCCS Ca' Granda-Maggiore Policlinico Hospital Foundation, Italy

${ }^{2}$ Dipartimento di Scienze Biomediche Chirurgiche e Odontoiatriche, Università degli Studi di Milano, Hematology and Transplantation Unit, IRCCS Ca' Granda-Maggiore Policlinico Hospital Foundation, Italy

${ }^{3}$ Laboratorio Centrale di Analisi Chimico Cliniche e Microbiologia, Fondazione IRCCS, Ca' Granda Ospedale Maggiore Policlinico, Milano, Italy

${ }^{4}$ Division of Haematology, Niguarda Hospital, Milan, Italy

"Corresponding author: Fracchiolla NS, Hematology and Transplantation Unit, IRCCS Ca' Granda-Maggiore Policlinico Hospital Foundation, Italy, Tel: +39 02-55033520; Fax: +39 02-55034182; E-mail: ns.fracchiolla@gmail.com

Received date: May 29, 2014, Accepted date: Sep 30, 2015, Publication date: Oct 05, 2015

Copyright: (c) 2015 Fracchiolla NS, et al. This is an open-access article distributed under the terms of the Creative Commons Attribution License, which permits unrestricted use, distribution, and reproduction in any medium, provided the original author and source are credited.

\section{Abstract}

Background: Oxidative status can be altered in cancer.

Objective: To evaluate the role of oxidative stress as plasma biomarker able to predict sepsis severity in oncohaematological patients experiencing infections after chemotherapy.

Methods: Spectrophotometric measurement of Reactive oxigen metabolites (ROMs) levels and Total antioxidant capacity (TAC) in 15 patients prospectively enrolled and 52 healthy volunteers.

Results: ROMs baseline values were higher in patients and raised during fever. Severe sepsis was associated with the highest degree of ROMs increase. TAC levels were always low, both at baseline and during infection.

Conclusions: Our results confirm oxidative status derangement during post-chemotherapy infections. ROMs concentrations could represent an easily detectable biomarker correlated with sepsis severity in post-chemotherapy neutropenia.

Keywords: Oxidative stress; Haematologic malignancies; Sepsis; Chemotherapy

\section{Introduction}

Haematological cancers and infections can be associated to alteration of oxidative status [1,2], that is defined as the balance between Reactive Oxygen Metabolites (ROMs) and Total Antioxidant Capacity (TAC) production. High-dose chemotherapy and haematopoietic stem cell transplantation (HSCT), employed for haematological malignancies, and contributes to ROS release [1]. The generation of reactive oxygen species appear to be the final mechanism in the activity of anticancer drugs, and is associated with a systemic oxidative stress $[3,4]$, that may be involved in the genesis of organ damage, as is in the case of anthracycline cardio toxicity [5].

The available data on oxidative status in haematological patients consist on limited series lacking sequential evaluation during diseaserelated events $[1,6]$. Sepsis, which is a leading cause of death in critically ill patients, is a complex inflammatory syndrome with a wide spectrum of severity. It is defined as infection associated with a systemic inflammatory response syndrome (SIRS) characterised by the presence of two or more of these conditions: body temperature $>38.3^{\circ} \mathrm{C}$ or $<36^{\circ} \mathrm{C}$, heart rate $>90$ beats $/ \mathrm{min}$ or $\mathrm{PaCO}_{2}<32 \mathrm{mmHg}$, white blood cell count $>12 \times 10^{9} / \mathrm{L}$ or $<4 \times 10^{9} / \mathrm{L}$. Severe sepsis occurs when organ dysfunction is associated. Multiple organ dysfunction syndrome (MODS) is defined as the simultaneous impairment of at least two organ systems. Septic shock is defined in adults as persisting hypotension despite adequate volume resuscitation [3].

The prevalent hypothesis regarding the mechanisms of sepsis and septic shock indicates that this complex condition is caused by an excessive defensive and inflammatory response to pathogens, characterised by massive increases in reactive oxygen species (ROMs), nitric oxide (NO) and inflammatory cytokines. The consequences of these events are systemic damage to the vascular endothelium, tissue hypooxygenation, glutathione depletion and mitochondrial respiratory dysfunction, with diminished levels of ATP and $\mathrm{O}_{2}$ consumption. This sequence of events, that leads to multi-organ failure and death, were extensively described during the final phase of septic shock [2,7-11]

Sepsis diagnosis and evaluation of its severity is complicated by the highly variable and non-specific nature of the signs and symptoms of this syndrome. However, the early diagnosis and stratification of the severity of sepsis is very important, increasing the possibility of starting timely and specific treatment. Biomarkers may have an important place in this process, because they may predict sepsis severity and can differentiate bacterial from viral and fungal infections, and systemic sepsis from local infection. Many biomarkers have been evaluated for use in sepsis. Most of them had been tested clinically, primarily as prognostic markers; relatively few have been used for diagnosis [12,13]. The leading cause of treatment-related mortality among patients with hematological malignancies undergoing 
Citation: Fracchiolla NS, Cortelezzi A, Orofino N, Novembrino C, De Giuseppe R, et al. (2015) Oxidative Stress as Marker of Sepsis in Onco Hematological Patients: A Pilot Study. J Blood Disord Transfus 6: 311. doi:10.4172/2155-9864.1000311

Page 2 of 5

chemotherapy is sepsis and multi-organ failure due to systemic infection during neutropenia. Most neutropenic patients with infections present with fever as the first and in many cases the only symptom. In addition, clinical signs may be unspecific and fever may due also to other causes. Microbiological diagnostic tests are not sufficiently rapid, sensitive or specific to identify the microbial causes of fever. For this reason many marker of infection have been searched for a rapid identification of an infectious event. For example, procalcitonin (PCT) has become popular as novel infection marker. The use of this analyte in onco-hematological patients has provided controversial results and no agreement exists about its capacity to differentiate infective fever from other inflammatory processes such as mucositis or graft versus host disease (GVHD) [14-16]. In this pilot study we investigated the oxidative status of haematological patients and correlated it with diagnosis, therapies and septic complications during the post-chemotherapy neutropenic phase.

\section{Methods}

ROMs levels and TAC were prospectively measured in 15 haematological patients (8M/7F, aged 25-65, median age 53), and compared with ROMs levels and TAC measured in 52 age-matched healthy controls ( 34 males, 18 females, aged 26-84 years, median age 50 years). The patients were treated with haemopoietic stem cell transplantation (HSCT; N=10, 6 autologus transplants, 4 allogeneic transplants), or high-dose chemotherapy $(\mathrm{N}=5)$ according to each patient's disease and clinical needs (Table 1).

Blood samples were collected on admission, before, during and after chemo/radiotherapy (days $0,+3,+6,+14,+21$ ) and then once weekly until discharge. In case of infections, blood samples were collected every three days until clinical resolution.

\begin{tabular}{|c|c|c|c|c|}
\hline \multicolumn{5}{|c|}{ Patients receiving haemopoietic stem cell transplantation } \\
\hline $\mathrm{Pt}$ & Age/sex & Diagnosis & Transplantation type & Conditioning regimen \\
\hline 1 & 60/F & MM IgAk & Autolougus & Melphalan $200 \mathrm{mg} / \mathrm{mq}$ \\
\hline 2 & $53 / \mathrm{F}$ & Mantle cell lymphoma & Autolougus & Melphalan $200 \mathrm{mg} / \mathrm{mq}$ \\
\hline 3 & $65 / F$ & MM IgG lambda & Autolougus & Melphalan $200 \mathrm{mg} / \mathrm{mq}$ \\
\hline 4 & $50 / M$ & MM IgG lambda & Autolougus & Melphalan $200 \mathrm{mg} / \mathrm{mq}$ \\
\hline 5 & $61 / M$ & ALL Ph'+ & Autolougus & Etoposide $75 \mathrm{mg} / \mathrm{mq}+6$-mercaptopurine $150 \mathrm{mg} / \mathrm{mq}$ \\
\hline 6 & $62 / F$ & MM IgAk & Autolougus & Melphalan $200 \mathrm{mg} / \mathrm{mq}$ \\
\hline 7 & $34 / \mathrm{M}$ & AML & Allogeneic & Cyclophosphamide + TBI 1200 cGy \\
\hline 8 & $25 / F$ & ALL & Allogeneic & Cyclophosphamide + Thiotepa \\
\hline 9 & $46 / M$ & Mycosis Fungoides & RIC allogeneic & Pentostatin + TBI 2 Gy \\
\hline 10 & $46 / M$ & Mycosis Fungoides & RIC allogeneic & Pentostatin + TBI 2 Gy \\
\hline \multicolumn{5}{|c|}{ Patients receiving chemotherapy } \\
\hline Pt & Age/sex & Diagnosis & \multicolumn{2}{|l|}{ Treatment } \\
\hline 11 & $59 / F$ & B-CLL & \multicolumn{2}{|l|}{ Chlorambucil + FCR } \\
\hline 12 & $54 / \mathrm{M}$ & AML & \multicolumn{2}{|l|}{ "MICE" scheme } \\
\hline 13 & $25 / F$ & ALL & \multicolumn{2}{|l|}{ "McMaster" scheme } \\
\hline 14 & $59 / M$ & AML & \multicolumn{2}{|l|}{ "FLANG" scheme } \\
\hline 15 & $48 / \mathrm{M}$ & ALL & \multicolumn{2}{|l|}{ "SPLIT" scheme } \\
\hline
\end{tabular}

Table 1: Patient's characteristics and therapies received.

Serum ROMs concentrations were measured by a spectrophotometric method using a commercial kit (dROMs test, Diacron International, Grosseto, Italy) on F.R.E.E. analyzer (Diacron) as previously described [17]. Serum TAC was measured by a spectrophotometric method using a commercial kit (OXY-Adsorbent test, Diacron International) on F.R.E.E. analyzer (Diacron International) as previously described [17].
Bacteraemia was defined by at least one positive blood culture.

Neutropenic fever (NF) was defined as a temperature of $\geq 38.3^{\circ} \mathrm{C}$ on several occasions in a patient whose neutrophil count is $<500 / \mu \mathrm{L}$ or is expected to fall to that level in 1-2 days.

Sepsis was defined as the presence of infection associated with a SIRS. 
Citation: Fracchiolla NS, Cortelezzi A, Orofino N, Novembrino C, De Giuseppe R, et al. (2015) Oxidative Stress as Marker of Sepsis in Onco Hematological Patients: A Pilot Study. J Blood Disord Transfus 6: 311. doi:10.4172/2155-9864.1000311

Page 3 of 5

We considered ROMs and TAC basal values, the maximum value reached during the clinical event, the difference $(\Delta$-Delta) between the value before the clinical event and the maximum value reached during the event and the values measured on days $+3,+6,+14$ and at discharge. The ROMs levels and TAC were compared with those of 52 healthy controls. The ROMs diagnostic relevance was tested for the (NF), bacteraemia and sepsis.

Sensibility and specificity levels have been recorded in a curve (ROC-Receiver-Operator Characteristic curve) according to their different values in order to identify the best cut-off level which can optimise both specificity and sensibility. Patients were initially divided into three groups according to therapy (autologus transplantation, allogeneic transplantation or conventional chemotherapy group) in order to assess whether there were significant differences between the values of ROMS and TAC in the three groups caused by different underlying disease and previous treatments.

In the first phase we compared the baseline values of each patient with the extreme value (maximum in case of ROMs and minimum value in case of TAC) recorded during the clinical event in the same patient, with the aim of assessing whether the absolute value of the analyte was a reliable indicator of ongoing inflammatory event.

We also evaluated ROMs and TAC variations during the course of the clinical event, comparing the value recorded before its onset with the maximum value reached during the same event. We compared the mean differences between the maximum value reached during fever, bacteraemia and sepsis and the baseline value before the clinical event, in order to asses whether it was possible to predict severity of the clinical events on the basis of the levels of analyte increase.

\section{Results}

Globally, 18 infectious complications were evaluated: 6 cases of bacteraemia, 6 of sepsis and 6 of (NF).

At baseline, ROMs levels and TAC did not differ subdividing patients on the basis of diagnosis. By comparing patient and healthy volunteers we showed that patients' baseline ROMs levels and TAC were significantly higher (385 vs 270 UCarr) and lower (280 vs 391 $\mu \mathrm{molHClO} / \mathrm{ml})$ respectively $(\mathrm{p}<0.001$, Mann-Whitney U-Test) than healthy controls' (Figure 1).

We did not show any statistically significant difference comparing ROMs and TAC values at base-line and at subsequent time points on days $+3,+6,+14(\mathrm{p}=\mathrm{ns})$ and at discharge $(\mathrm{p}=\mathrm{ns})$ in patient receiving different treatments (chemotherapy alone, autologous or allogeneic transplantation).
Maximal ROMs values (M-ROMs) registered during sepsis were significantly higher than basal values ( $\mathrm{p}<0.05$, Wilcoxon Test), while during bacteraemia and NF, M-ROMs did not differ from basal values ( $\mathrm{p}=\mathrm{ns})$.

$\Delta$-ROMs values were significantly higher during sepsis than during bacteraemia and NF $(\mathrm{p}<0.05$ and $\mathrm{p}<0.01$ Mann Whitney U-test, respectively) (Table 2).

ROMs predictive ability as a marker of sepsis was verified by analysing the ROC curve. Setting a cut-off value of 364 Ucarr, the area under the curve was 0.769 when ROMs was used to discriminate sepsis (sensitivity $88.2 \%$; specificity $59.4 \%$ ) (Figure 2).

When comparing TAC minimal values registered during a clinical event and TAC basal values, no significant differences were found between the two antioxidant capacities either in NF, bacteraemia or sepsis cases.

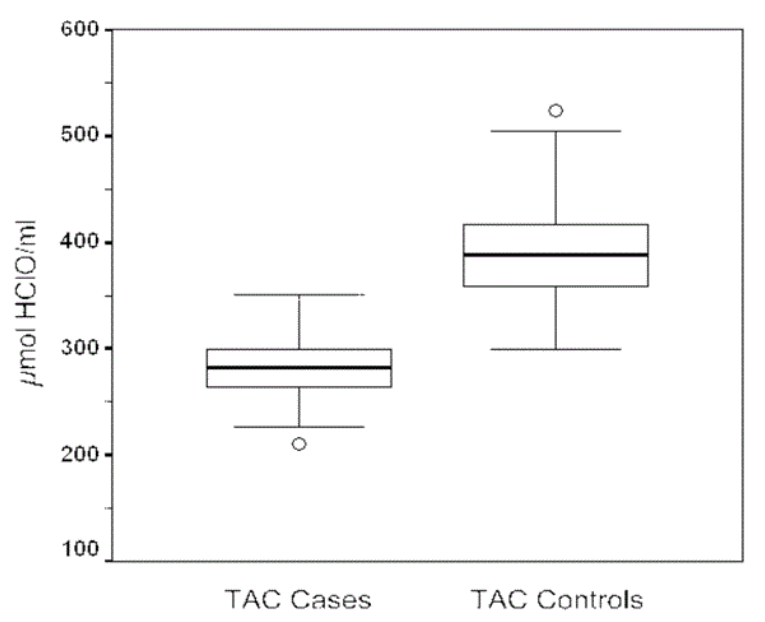

Figure 1: Basal M-ROMs (a) and m-TAC (b) levels depicted as boxplots of hematological patients (Cases) and healthy controls (Controls). Each box-plot depicts the median (middle line), the values from the upper to lower quartiles (central box) and the upper/lower quartile $\pm 1.5 \times$ interquartile range (horizontal lines) of one category. Outliers (i.e. values outside these borders) are depicted as circles $\mathrm{p}<0.001$.

\begin{tabular}{|l|l|l|l|l|l|}
\hline Infective Episodes & Tot & $\begin{array}{l}\text { median Maximal ROMs concentration } \\
\text { UCarr } \\
\text { (range) }\end{array}$ & $\begin{array}{l}\text { median } \Delta-\text { ROMs } \\
\text { UCarr } \\
\text { (range) }\end{array}$ & $\begin{array}{l}\text { median minimum TAC concentration } \\
\mu \text { mol HCIO/mL (range) }\end{array}$ & $\begin{array}{l}\text { median d-TAC } \\
\mu m o l H C I O / m L \\
\text { (range) }\end{array}$ \\
\hline NF & 6 & 453 & 66 & 245 & -14 \\
\hline & & $(342-622)$ & $(21-116)$ & $(218-283)$ & $(0-37)$ \\
\hline Bacteraemia & 6 & 403 & 48 & 276 & -21 \\
\hline & & $(309-514)$ & $(9-68)$ & $(205-369)$ & $(0-41)$ \\
\hline Sepsis & 6 & $542^{*}$ & $136^{\S}$ & 307 & -31 \\
\hline
\end{tabular}




\begin{tabular}{|l|l|l|l|l|}
\hline & $(390-704)$ & $(68-234)$ & $(251-383)$ & $(0-50)$ \\
\hline \multicolumn{2}{|l|}{$p<0.05$ vs baseline (Wilcoxon's test); $\$ \mathrm{p}<0.05$ vs FUO and ${ }^{\circ} \mathrm{p}<0.01$ vs bacteraemia (Man-Whitney U-Test). } \\
\hline
\end{tabular}

Table 2: ROMs and TAC levels.

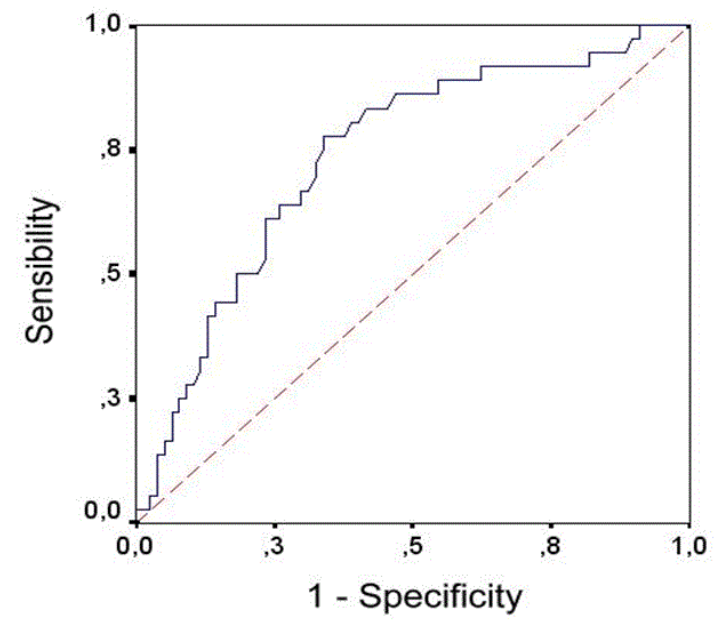

Figure 2: ROC curve of ROMs.

\section{Discussion and Conclusions}

This pilot study showed that our haematological patients had an impaired oxidative status due to high ROMs levels and low TAC.

Chemotherapy and haematopoietic stem cell transplantation (HSCT) are effective therapies for hematological tumours. Most malignancies are associated with an excess production of oxidative stress. Mazor et al. assessed the antioxidant levels in plasma of seven children affected by acute lymphoblastic leukemia (ALL) and compared the results with those obtained in six children treated for different solid tumors and lymphomas, showing that the higher oxidative stress of children with ALL is associated with the therapeutic protocol used to treat this malignancy which is more aggressive than that used for solid tumors [18-20].

Febrile neutropenia in patients with haematologic malignancies undergoing chemotherapy, is characterized by an increased susceptibility to infectious complications, and a higher risk of septic shock with a relevant mortality rate. Furthermore most neutropenic patients with infection present fever as the first symptom and the clinical course of neutropenic fever is extremely heterogeneous, ranging from severe sepsis and septic shock to mild diseases. Although methods for predicting the development of severe NF could help to reduce treatment-related mortality, early markers capable to predict its outcome have not yet been established.

Many analytical parameters are under investigation in this setting. For example, procalcitonin (PCT) has become popular as novel infection marker. Its use in onco-haematological patients has provided controversial results and no agreement exists about its capacity to differentiate infective fever from other inflammatory processes such as mucositis or graft versus host disease (GVHD) [14-16]. Furthermore the peak value of PCT, like the peak value of C-Reactive Protein, is often reached days after the onset of NF.

It has been already shown that plasma red-ox status correlates to severity scores in critically ill patients in intensive care unit [21-23]. Several researchers have also reported that ROS increases in severe sepsis both in patients and animal models, compared with non-critical sepsis or controls, respectively. However, the kinetics of ROS and antioxidants in neutropoenic patients remain unclear, especially in patients with NF [24-26].

In 2010 Nishikawa et al. investigated the association between ROMs levels and the severity of NF in a small series of pediatric oncohaematologic patients, showing that levels of ROMs in NF patients with SIRS were significantly lower than those in subjects without SIRS and they concluded that low ROMs level at fever onset is associated with severe clinical features [27].

On the contrary, in our series, at the moment of first oxidative status assessment, patients had been pretreated with heterogeneous chemotherapeutic regimens, and showed significantly higher ROMs levels and lower antioxidant capacity in comparison to healthy controls. Furthermore, the patient's impaired oxidative status did not improve after the end of therapy, suggesting a state of chronic oxidative stress, to which both the underlying disease and the treatments received, might have contributed.

In case of infectious event during the neutropenic period after chemotherapy, our cases showed a further increase in ROMs level compared to baseline, coupled with the persistence of an impaired low antioxidant capacity, as measured by TAC. In particular we noted that only in case of subsequent development of sepsis there was a significant elevation of ROMs levels, suggesting that their serial measurements (easily detectable in serum) could be a useful biomarker of severity infection in neutropenic onco-hermatological patient.

It is well known that the allmark of sepsis is a dysregulated inflammatory response, characterized by massive cytokine release, oxidative stress and mitochondrial disfunction. During sepsis and septic shock, antioxidant defenses are overwhelmed, and ROMs cause cellular damage, contributing to organ dysfunction. Our patients showed a constantly low antioxidant capacity when compared with healthy people, indicating a major susceptibility of oxidative-induced organ damage during an infectious event. Studies on animal models have demonstrated that antioxidant therapies, in particular those acting preferentially in mitochondria, reduce mitochondrial damage and organ dysfunction and decrease inflammatory responses in a rat model of acute sepsis [28].

In conclusion, the observations of our pilot study, as well as providing a valuable marker of inflammation, complementary to those routinely used, could represent the biological basis for antioxidant support therapy during a septic episode in neutropenic patient. 
Citation: Fracchiolla NS, Cortelezzi A, Orofino N, Novembrino C, De Giuseppe R, et al. (2015) Oxidative Stress as Marker of Sepsis in Onco Hematological Patients: A Pilot Study. J Blood Disord Transfus 6: 311. doi:10.4172/2155-9864.1000311

Page 5 of 5

\section{Acknowledgments}

The authors report no conflicts of interest.

\section{References}

1. Battisti V, Maders LD, Bagatini MD, Santos KF, Spanevello RM, et al. (2008) Measurement of oxidative stress and antioxidant status in acute lymphoblastic leukemia patients. Clin Biochem 41: 511-518.

2. Víctor VM, Espulgues JV, Hernández-Mijares A, Rocha M (2009) Oxidative stress and mitochondrial dysfunction in sepsis: a potentia therapy with mitochondria-targeted antioxidants. Infect Disord Drug Targets 9: 376-389.

3. Levy MM, Fink MP, Marshall JC, Abraham E, Angus D, et al. (2003) 2001 SCCM/ESICM/ACCP/ATS/SIS International Sepsis Definitions Conference. Crit Care Med 31: 1250-1256.

4. Schumacker PT (2006) Reactive oxygen species in cancer cells: live by the sword, die by the sword. Cancer Cell 10: 175-176.

5. Simunek T, Sterba M, Popelova O, Adamcova M, Hrdina R, et al. (2009) Anthracycline-induced cardiotoxicity: overview of studies examining the roles of oxidative stress and free cellular iron. Pharmacol Rep 61: 154-171.

6. Alves B, Silmara A, Aranha FJ, Siegl TF, Souza CA, et al. (2010) Imbalances in serum angiopoietin concentrations are early predictors of septic shock development in patients with post chemotherapy febrile neutropenia. BMC Infectious disease 10: 143-151.

7. Andrades ME, Ritter C, Dal-Pizzol F (2009) The role of free radicals in sepsis development. Front Biosci (Elite Ed) 1: 277-287.

8. Victor VM, Rocha M, Esplugues JV, De la Fuente M (2005) Role of free radicals in sepsis: antioxidant therapy. Curr Pharm Des 11: 3141-3158.

9. Victor VM, Rocha M, De la Fuente M (2004) Immune cells: free radicals and antioxidants in sepsis. Int Immunopharmacol 4: 327-347.

10. Hotchkiss RS, Karl IE (2003) The pathophysiology and treatment of sepsis. N Engl J Med 348: 138-150.

11. Victor VM, De la Fuente M (2003) Immune cells redox state from mice with endotoxin-induced oxidative stress. Involvement of NF-kappaB. Free Radic Res 37: 19-27.

12. Borrelli E, Roux-Lombard P, Grau GE, Girardin E, Ricou B, et al. (1996) Plasma concentrations of cytokines, their soluble receptors, and antioxidant vitamins can predict the development of multiple organ failure in patients at risk. Crit Care Med 24: 392-397.

13. Charalampos P, Vincent JL (2010) Sepsis biomarkers: a review. Crit Care14: R15.

14. Schüttrumpf S, Binder L, Hagemann T, Berkovic D, Trümper L, et al. (2003) Procalcitonin: a useful discriminator between febrile conditions of different origin in hemato-oncological patients? Ann Hematol 82: 98-103.
15. Sakr Y, Sponholz C, Tuche F, Brunkhorst F, Reinhart K (2008) The role of procalcitonin in febrile neutropenic patients: review of the literature. Infection 36: 396-407.

16. Sarmati L, Beltrame A, Dori L, Maffongelli G, Cudillo L, et al. (2010) Procalcitonin is a reliable marker of severe systemic infection in neutropenic haematological patients with mucositis. Am J Hematol 85: 380-383.

17. Campise M, Bamonti F, Novembrino C, Ippolito S, Tarantino A, et al. (2003) Oxidative stress in kidney transplant patients. Transplantation 76 1474-1478.

18. Al-Gayyar MM, Eissa LA, Rabie AM, El-Gayar AM (2007) Measurements of oxidative stress status and antioxidant activity in chronic leukaemia patients. J Pharm Pharmacol 59: 409-417.

19. Mazor D, Abucoider A, Meyerstein N, Kapelushnik J (2008) Antioxidant status in pediatric acute lymphocytic leukemia (ALL) and solid tumors: the impact of oxidative stress. Pediatr Blood Cancer 51: 613-615.

20. Chevion S, Or R, Berry EM (1999) The antioxidant status of patients subjected to total body irradiation. Biochem Mol Biol Int 47: 1019-1027.

21. Alonso de Vega JM, Díaz J, Serrano E, Carbonell LF (2000) Plasma redox status relates to severity in critically ill patients. Crit Care Med 28: 1812-1814.

22. Hoesel LM, Neff TA, Neff SB, Younger JG, Olle EW, et al. (2005) Harmful and protective roles of neutrophils in sepsis. Shock 24: 40-47.

23. Goode HF, Cowley HC, Walker BE, Howdle PD, Webster NR (1995) Decreased antioxidant status and increased lipid peroxidation in patients with septic shock and secondary organ dysfunction. Crit Care Med 23: 646-651.

24. Gutteridge JM, Mitchell J (1999) Redox imbalance in the critically ill. Br Med Bull 55: 49-75.

25. Gitto E, Karbownik M, Reiter RJ, Tan DX, Cuzzocrea S, et al. (2001) Effects of melatonin treatment in septic newborns. Pediatr Res 50: 756-760.

26. Pascual C, Karzai W, Meier-Hellmann A, Oberhoffer M, Horn A, et al. (1998) Total plasma antioxidant capacity is not always decreased in sepsis. Crit Care Med 26: 705-709.

27. Nishikawa T, Okamoto Y, Kodama Y, Tanabe T, Shinkoda Y, et al. (2010) Serum derivative of reactive oxygen metabolites (d-ROMs) in pediatric hemato-oncological patients with neutropenic fever. Pediatr Blood Cancer 55: 91-94.

28. Lowes DA, Webster NR, Murphy MP, Galley HF (2013) Antioxidants that protect mitochondria reduce interleukin- 6 and oxidative stress, improve mitochondrial function, and reduce biochemical markers of organ dysfunction in a rat model of acute sepsis. Br J Anaesth 110: 472-480. 